\title{
Article
}

\section{On Killing Vector Fields on Riemannian Manifolds}

\author{
Sharief Deshmukh ${ }^{1}(\mathbb{D}$ and Olga Belova $2, *$ (]) \\ 1 Department of Mathematics, College of Science, King Saud University, P.O. Box-2455, \\ Riyadh 11451, Saudi Arabia; shariefd@ksu.edu.sa \\ 2 Institute of Physical and Mathematical Sciences and IT, Immanuel Kant Baltic Federal University, \\ A. Nevsky Str. 14, 236016 Kaliningrad, Russia \\ * Correspondence: olgaobelova@mail.ru; Tel.: +7-921-610-5949
}

check for updates

Citation: Deshmukh, S.; Belova, O. On Killing Vector Fields on

Riemannian Manifolds. Mathematics 2021, 9, 259. https://doi.org/ $10.3390 /$ math 9030259

Academic Editor: Pablo Sebastián Alegre Rueda

Received: 14 January 2021

Accepted: 25 January 2021

Published: 28 January 2021

Publisher's Note: MDPI stays neutral with regard to jurisdictional clai$\mathrm{ms}$ in published maps and institutional affiliations.

Copyright: $\odot 2021$ by the authors. Licensee MDPI, Basel, Switzerland. This article is an open access article distributed under the terms and conditions of the Creative Commons Attribution (CC BY) license (https:// creativecommons.org/licenses/by/ $4.0 /)$.

\begin{abstract}
We study the influence of a unit Killing vector field on geometry of Riemannian manifolds. For given a unit Killing vector field $\mathbf{w}$ on a connected Riemannian manifold $(M, g)$ we show that for each non-constant smooth function $f \in C^{\infty}(M)$ there exists a non-zero vector field $\mathbf{w}^{f}$ associated with $f$. In particular, we show that for an eigenfunction $f$ of the Laplace operator on an $n$-dimensional compact Riemannian manifold $(M, g)$ with an appropriate lower bound on the integral of the Ricci curvature $S\left(\mathbf{w}^{f}, \mathbf{w}^{f}\right)$ gives a characterization of the odd-dimensional unit sphere $\mathbf{S}^{2 m+1}$. Also, we show on an $n$-dimensional compact Riemannian manifold $(M, g)$ that if there exists a positive constant $c$ and non-constant smooth function $f$ that is eigenfunction of the Laplace operator with eigenvalue $n c$ and the unit Killing vector field $\mathbf{w}$ satisfying $\|\nabla \mathbf{w}\|^{2} \leq(n-1) c$ and Ricci curvature in the direction of the vector field $\nabla f-\mathbf{w}$ is bounded below by $(n-1) c$ is necessary and sufficient for $(M, g)$ to be isometric to the sphere $\mathbf{S}^{2 m+1}(c)$. Finally, we show that the presence of a unit Killing vector field $\mathbf{w}$ on an $n$-dimensional Riemannian manifold $(M, g)$ with sectional curvatures of plane sections containing $\mathbf{w}$ equal to 1 forces dimension $n$ to be odd and that the Riemannian manifold $(M, g)$ becomes a K-contact manifold. We also show that if in addition $(M, g)$ is complete and the Ricci operator satisfies Codazzi-type equation, then $(M, g)$ is an Einstein Sasakian manifold.
\end{abstract}

Keywords: killing vector field; K-contact manifold; sasakian manifold; Einstein-Sasakian manifold

\section{Introduction}

Killing vector fields are known to play vital role in influencing the geometry as well as topology of Riemannian manifolds (see [1-10]) and being incompressible fields play important role in physics (see [11]). If we restrict the length of a Killing vector fields such as constant length, then it severely restricts the geometry of Riemannian manifolds on which they are set. For instance, there are no unit Killing vector fields on even-dimensional spheres $\mathbf{S}^{2 n}(c)$. However, there are unit Killing vector fields on odd-dimensional spheres $\mathbf{S}^{2 n+1}(c)$ as well on odd-dimensional ellipsoids (see $\left.[4,12,13]\right)$. Most importantly, on all Kcontact manifolds there is a unit Killing vector field called the Reeb vector field (see [12,13]). There are other important structures and special vector fields, which also influence the geometry of a Riemannian manifold (see [14]).

In this paper, we are interested in studying the impact of presence of a unit Killing vector field $\mathbf{w}$ on the geometry of a connected Riemannian manifold $(M, g)$. Our first interesting finding is that for each smooth non-constant function $f$ on $M$, there is naturally associated a non-zero vector field $\mathbf{w}^{f}$ on $M$ that is orthogonal to $\mathbf{w}$ (see Proposition 1 ). Then we consider the associated vector field $\mathbf{w}^{f}$ corresponding to eigenfunction $f$ of Laplace operator on a compact Riemannian manifold $(M, g)$ corresponding to eigenvalue $\operatorname{dim} M$ and show that if the integral of Ricci curvature in the direction of $\mathbf{w}^{f}$ is bounded below by certain bound, forces $\operatorname{dim} M$ to be odd and thus gives a necessary and sufficient for $(M, g)$ to be isometric to the unit sphere $\mathbf{S}^{2 m+1}$ (see Theorem 1). A similar characterization of the sphere $\mathbf{S}^{2 m+1}(c)$ using the eigenfunction $f$ on a compact $n$-dimensional $(M, g)$ with eigenvalue $n c$ is obtained (see Theorem 2 ). 
Given a unit Killing vector field $\mathbf{w}$ on a connected Riemannian manifold $(M, g)$ there is associated skew-symmetric tensor field $\Psi$ given by the covariant derivative of $\mathbf{w}$, giving a structure $(\Psi, \mathbf{w}, \alpha, g)$ on $M$, where $\alpha$ is 1 -form dual to $\mathbf{w}$. A natural question is under which situation the structure $(\Psi, \mathbf{w}, \alpha, g)$ becomes a K-contact structure? We answer this question and find a necessary and sufficient condition in terms of sectional curvatures of plane sections containing $\mathbf{w}$ (see Theorem 3 ). We also find conditions under which a Riemannian manifold admitting a unit Killing vector field is an Einstein manifold (see Theorems 4 and 5). Finally, as a by-product of Theorem 3 with an additional condition that the Ricci operator is Codazzi type tensor on a Riemannian manifold $(M, g)$ that admits a unit Killing vector field $\mathbf{w}$, we show that $(M, g)$ is an Einstein Sasakian manifold (see Corollary 2).

\section{Preliminaries}

Recall that a vector field $\mathbf{w}$ on a Riemannian manifold $(M, g)$ is said to be a Killing vector fields if

$$
£_{\mathbf{w}} g=0,
$$

where $£_{\mathbf{w}}$ being Lie-derivative of metric $g$ with respect to $\xi$; or equivalently,

$$
g\left(\nabla_{X_{1}} \mathbf{w}, X_{2}\right)=-g\left(\nabla_{X_{2}} \mathbf{w}, X_{1}\right), \quad X_{1}, X_{2} \in \mathfrak{X}(M),
$$

where $\nabla$ is the Riemannian connection on $(M, g)$ and $\mathfrak{X}(M)$ is Lie-algebra of smooth vector fields on $M$. Please note that a parallel vector field is a Killing vector field, we say a Killing vector field is a non-trivial Killing vector field if it is not parallel. For the Killing vector field $\mathbf{w}$ on an $n$-dimensional Riemannian manifold $(M, g)$, we denote by $\alpha$ the smooth 1 -form dual to $\mathbf{w}$, i.e., $\alpha(X)=g(\mathbf{w}, X), X \in \mathfrak{X}(M)$. In addition, we define a skew-symmetric (1.1)-tensor field $\Psi$ by

$$
g\left(\Psi X_{1}, X_{2}\right)=\frac{1}{2} d \alpha\left(X_{1}, X_{2}\right)=\frac{1}{2} g\left(\nabla_{X_{1}} \mathbf{w}, X_{2}\right)-\frac{1}{2} g\left(\nabla_{X_{2}} \mathbf{w}, X_{1}\right), \quad X_{1}, X_{2} \in \mathfrak{X}(M) .
$$

Then, using Equation (1), it follows that

$$
g\left(\nabla_{X_{1}} \mathbf{w}, X_{2}\right)=g\left(\Psi X_{1}, X_{2}\right), \quad X_{1}, X_{2} \in \mathfrak{X}(M),
$$

that is,

$$
\nabla_{X} \mathbf{w}=\Psi X, \quad X \in \mathfrak{X}(M) .
$$

The curvature tensor field $R$ and the Ricci tensor $S$ of $(M, g)$ are given by

$$
R\left(X_{1}, X_{2}\right) X_{3}=\left[\nabla_{X_{1}}, \nabla_{X_{2}}\right] X_{3}-\nabla_{\left[X_{1}, X_{2}\right]} X_{3}
$$

and

$$
S\left(X_{1}, X_{2}\right)=\sum_{i=1}^{n} g\left(R\left(e_{i}, X_{1}\right) X_{2}, e_{i}\right),
$$

respectively, where $\left\{e_{1}, . ., e_{n}\right\}$ is a local orthonormal frame on $M$.

According to symmetry of the Ricci tensor $S$, we get a symmetric operator $T$ called the Ricci operator of $M$ and defined by

$$
g\left(T X_{1}, X_{2}\right)=S\left(X_{1}, X_{2}\right), \quad X_{1}, X_{2} \in \mathfrak{X}(M) .
$$

The scalar curvature $\tau$ of $M$ is given by $\tau=\operatorname{Tr} T$. Please note that $\nabla \tau$ - the gradient of the scalar curvature $\tau$-satisfies

$$
\frac{1}{2} \nabla \tau=\sum_{i=1}^{n}(\nabla T)\left(e_{i}, e_{i}\right),
$$


where $\nabla T$ is the covariant derivative of $T$, defined by

$$
(\nabla T)\left(X_{1}, X_{2}\right)=\nabla_{X_{1}} T X_{2}-T \nabla_{X_{1}} X_{2}
$$

Please note that if $\mathbf{w}$ is a Killing vector field on a Riemannian manifold $(M, g)$, then using Equations (2) and (3), we have

$$
R\left(X_{1}, X_{2}\right) \mathbf{w}=(\nabla \Psi)\left(X_{1}, X_{2}\right)-(\nabla \Psi)\left(X_{2}, X_{1}\right), \quad X_{1}, X_{2} \in \mathfrak{X}(M) .
$$

From definition of $\Psi$, we see that the smooth 2-form $g\left(\Psi X_{1}, X_{2}\right)$ is closed and as a result of using Equation (5), we make a conclusion

$$
(\nabla \Psi)\left(X_{1}, X_{2}\right)=R\left(X_{1}, \mathbf{w}\right) X_{2}, \quad X_{1}, X_{2} \in \mathfrak{X}(M) .
$$

Observe that if $\mathbf{w}$ is a Killing vector field of constant length on a Riemannian manifold $(M, g)$, then taking the inner product with $\mathbf{w}$ in Equation (2), we get $g(\Psi X, \mathbf{w})=0$, $X \in \mathfrak{X}(M)$, and as $\Psi$ is skew-symmetric, we conclude

$$
\Psi \mathbf{w}=0 .
$$

For given a smooth function $f$ on a Riemannian manifold the Hessian operator $\mathcal{H}_{f}$ is defined by

$$
\mathcal{H}_{f}(X)=\nabla_{X} \nabla f, \quad X \in \mathfrak{X}(M),
$$

which is a symmetric operator with

$$
\operatorname{Tr} \mathcal{H}_{f}=\Delta f,
$$

where $\Delta$ is the Laplace operator. The Hessian $\operatorname{Hess}(f)$ of $f$ is defined by

$$
\operatorname{Hess}(f)\left(X_{1}, X_{2}\right)=g\left(\mathcal{H}_{f}\left(X_{1}\right), X_{2}\right), \quad X_{1}, X_{2} \in \mathfrak{X}(M) .
$$

Let $\mathbf{w}$ be a Killing vector field on a Riemannian manifold $(M, g)$ and $C^{\infty}(M)$ be the algebra of smooth functions on $M$. Then, using Equation (2) and skew-symmetry of the operator $\Psi$, it follows that $\operatorname{div} \mathbf{w}=0$ and that for a smooth function $f \in C^{\infty}(M)$, we have $\operatorname{div}(f \mathbf{w})=\mathbf{w}(f)$. Thus, we get

Lemma 1. Let $\mathbf{w}$ be a unit Killing vector field on a compact Riemannian manifold $(M, g)$. Then for a smooth function $f \in C^{\infty}(M)$

$$
\int_{M} \mathbf{w}(f)=0
$$

Lemma 2. Let $\mathbf{w}$ be a unit Killing vector field on a Riemannian manifold $(M, g)$. Then

$$
S(\mathbf{w}, \mathbf{w})=\|\Psi\|^{2} .
$$

Proof. Assume that $\mathbf{w}$ is a unit Killing vector field on a Riemannian manifold $(M, g)$. Then, using Equations (2) and (7), we have

$$
(\nabla \Psi)(X, \mathbf{w})=-\Psi^{2} X, \quad X \in \mathfrak{X}(M) .
$$

Now, using a local orthonormal frame $\left\{e_{1}, \ldots, e_{n}\right\}, n=\operatorname{dim} M$, and noting that

$$
T(\mathbf{w})=\sum_{i=1}^{n} R\left(\mathbf{w}, e_{i}\right) e_{i}
$$


and in view of Equation (6), we conclude

$$
T(\mathbf{w})=-\sum_{i=1}^{n}(\nabla \Psi)\left(e_{i}, e_{i}\right) .
$$

Taking the inner product in above equation with $\mathbf{w}$ and using Equation (9), we deduce

$$
S(\mathbf{w}, \mathbf{w})=\sum_{i=1}^{n} g\left(e_{i},-\Psi^{2} e_{i}\right)=\sum_{i=1}^{n} g\left(\Psi e_{i}, \Psi e_{i}\right)=\|\Psi\|^{2} .
$$

Lemma 3. Let $\mathbf{w}$ be a unit Killing vector field on a compact Riemannian manifold $(M, g)$. Then for a smooth function $f \in C^{\infty}(M)$

$$
\int_{M} S(\nabla f, \mathbf{w})=0 .
$$

Proof. Please note that the flow of the Killing vector field $\mathbf{w}$ consists of isometries of the Riemannian manifold, therefore, we have $\mathbf{w}(\boldsymbol{\tau})=0$. We use Equation (2) in computing the divergence $\operatorname{div}(T \mathbf{w})$ and get

$$
\begin{gathered}
\operatorname{div}(T \mathbf{w})=\sum_{i=1}^{n}\left((\nabla T)\left(\mathbf{w}, e_{i}\right)+T\left(\Psi e_{i}\right), e_{i}\right) \\
=g\left(\mathbf{w}, \sum_{i=1}^{n}(\nabla T)\left(e_{i}, e_{i}\right)\right)+\operatorname{Tr}(T \circ \Psi),
\end{gathered}
$$

where $\left\{e_{1}, \ldots, e_{n}\right\}$ is a local orthonormal frame, $n=\operatorname{dim} M$. Owing to symmetry of $T$ and skew-symmetry of $\Psi$, it follows that $\operatorname{Tr}(T \circ \Psi)=0$ and using Equation (4), we conclude $\operatorname{div}(T \mathbf{w})=0$. Also, we have

$$
\operatorname{div}(f T \mathbf{w})=S(\nabla f, \mathbf{w})+f \operatorname{div}(T \mathbf{w})=S(\nabla f, \mathbf{w}) .
$$

By integrating above equation, the desired result follows.

As a consequence of Lemma 2, we conclude the following

Corollary 1. On a hyperbolic space or a Euclidean space, there does not exists a non-trivial unit Killing vector field.

It is well known that the odd-dimensional unit sphere $\mathbf{S}^{2 m+1}$ possesses a unit Killing vector field $\xi$, the Reeb vector field provided by the Sasakian structure $(\varphi, \xi, \eta, g)$ on $\mathbf{S}^{2 m+1}$ (cf. [12]). In the rest of this section, we shall construct the unit Killing vector field on the sphere $\mathbf{S}^{2 m+1}(c), c>0$ and on odd-dimensional ellipsoids. Treating $\mathbf{S}^{2 m+1}(c)$ as real hypersurface of the complex manifold $\left(\mathbf{C}^{m+1}, J,\langle\rangle,\right)$ with unit normal $\zeta$ and shape operator $A=-\sqrt{c} I$, where $J$ is the complex structure and $\langle$,$\rangle is the Euclidean Hermitian metric$ on $\mathbf{C}^{m+1}$. We denote the Euclidean connection on $\mathbf{C}^{m+1}$ by $D$ and the induced metric and induce Riemannian connection on $\mathbf{S}^{2 m+1}(c)$ by $g$ and $\nabla$, respectively. Now, define a unit vector field $\mathbf{w}=-J \zeta$ on the sphere $\mathbf{S}^{2 m+1}(c)$. Then taking covariant derivative in the equation $\mathbf{w}=-J \zeta$ with respect to $X \in \mathfrak{X}\left(\mathbf{S}^{2 m+1}(c)\right)$ applying Gauss-Weingarten formulas for hypersurface, we conclude

$$
\nabla_{X} \mathbf{w}-\sqrt{c} g(X, \mathbf{w}) \zeta=-\sqrt{c} J X .
$$


Define an operator $\Psi$ on $S^{2 m+1}(c)$ by $\Psi X=-\sqrt{c}(J X)^{T}$, where $(J X)^{T}$ is the tangential projection of $J X$ on $S^{2 m+1}(c)$. Then, it follows that $\Psi$ is skew-symmetric operator and above equation on equating tangential and normal components gives

$$
\nabla_{X} \mathbf{w}=\Psi X, \quad X \in \mathfrak{X}\left(\mathbf{S}^{2 m+1}(c)\right)
$$

and it follows that $\mathbf{w}$ is a unit Killing vector field on the sphere $\mathbf{S}^{2 m+1}(c)$. Moreover, if we take a constant unit vector $\vec{b}$ on the complex space $C^{m+1}$ and define a smooth function $f=\langle\vec{b}, \zeta\rangle$ and letting $\mathbf{u}$ be the tangential projection of the vector $\vec{b}$ on the sphere $\mathbf{S}^{2 m+1}(c)$, we get

$$
\vec{b}=\mathbf{u}+f \zeta
$$

Taking covariant derivative in above equation with respect to $X \in \mathfrak{X}\left(\mathbf{S}^{2 m+1}(c)\right)$ and using Gauss-Weingarten formulas for hypersurface and noting that $D_{X} \vec{b}=0$, we obtain

$$
\nabla_{X} \mathbf{u}=-\sqrt{c} f X, \quad \nabla f=\sqrt{c} \mathbf{u}, \quad X \in \mathfrak{X}\left(\mathbf{S}^{2 m+1}(c)\right),
$$

that is,

$$
\nabla_{X} \nabla f=-c f X, \quad X \in \mathfrak{X}\left(\mathbf{S}^{2 m+1}(c)\right),
$$

where $\nabla f$ is the gradient of the smooth function $f$. We claim that $f$ is non-constant, for if $f$ is constant, then Equation (10) will imply $\mathbf{u}=0$ and $f=0$ and that $\vec{b}=0$, contrary to the fact that $\vec{b}$ is a unit vector field. Hence, $f$ is a non-constant function and Equation (11) implies that

$$
\Delta f=-(2 m+1) c f
$$

where $\Delta f=\operatorname{div} \nabla f$ is the Laplace operator acting on $f$.

Consider a smooth function $h: \mathbf{E}^{2 m+2} \rightarrow \mathbf{R}$, defined by

$$
h(u)=\sum_{i=1}^{m+1} u_{i}^{2}+a \sum_{i=m+2}^{2 m+2} u_{i}^{2}-1
$$

where $\mathbf{E}^{2 m+2}$ is the Euclidean space and the constant $a>0$,

$$
u=\left(u_{1}, \ldots, u_{m+1}, u_{m+2}, \ldots, u_{2 m+2}\right) \in \mathbf{E}^{2 m+2} .
$$

Then $M=h^{-1}(\{0\})$ is $(2 m+1)$-dimensional compact hypersurface of the Euclidean space $\mathbf{E}^{2 m+2}$. Let $g$ be the induced metric on the Ellipsoid $M$ as hypersurface of $\mathbf{E}^{2 m+2}$. Consider the vector field $\mathbf{w}$ on the Euclidean space $\mathbf{E}^{2 m+2}$

$$
\mathbf{w}=\left(-u_{2}, u_{1}, \ldots,-u_{m+1}, u_{m},-\sqrt{a} u_{m+3}, \sqrt{a} u_{m+2}, \ldots,-\sqrt{a} u_{2 m+2}, \sqrt{a} u_{2 m+1}\right),
$$

it follows that $\mathbf{w}$ is a Killing vector field on the Euclidean space $\mathbf{E}^{2 m+2}$ and its flow consists of isometries of the Euclidean space $\mathbf{E}^{2 m+2}$. Please note that length of $\mathbf{w}$ is non-constant on the Euclidean space $\mathbf{E}^{2 m+2}$. The function $h$ is invariant under flow $\left\{\phi_{t}\right\}($ as $\mathbf{w}(h)=0)$ of $\mathbf{w}$ and the ellipsoid is invariant under the flow $\left\{\phi_{t}\right\}$, consequently, the vector field $\mathbf{w}$ is tangent to the hypersurface $M$ and with respect the induced metric $g$, we have $g(\mathbf{w}, \mathbf{w})=1$, i.e., $\mathbf{w}$ is a unit Killing vector field on the compact Riemannian manifold $(M, g)$.

\section{Characterizations of Spheres}

In this section, we use unit Killing vector fields on an $n$-dimensional compact Riemannian manifold $(M, g)$ in finding two characterizations of the spheres. First, given a unit Killing vector field $\mathbf{w}$ on a connected Riemannian manifold $(M, g)$, for each smooth non-constant function $f \in C^{\infty}(M)$, we define a vector field $\mathbf{w}^{f}$ by

$$
\mathbf{w}^{f}=\nabla f-\mathbf{w}(f) \mathbf{w}
$$


and call $\mathbf{w}^{f}$ the associated vector field of $f$ with respect to $\mathbf{w}$. Please note that the associated vector field $\mathbf{w}^{f}$ is orthogonal to the unit Killing vector field $\mathbf{w}$. It is interesting to note that if $\mathbf{w}$ is non-trivial unit Killing vector field, then for each $f \in C^{\infty}(M)$, the associated vector field $\mathbf{w}^{f}$ is non-zero as seen in the following.

Proposition 1. Let $\mathbf{w}$ be a unit non-trivial Killing vector field on a connected Riemannian manifold $(M, g)$. Then for each non-constant function $f \in C^{\infty}(M)$ the associated vector field $\mathbf{w}^{f}$ is non-zero.

Proof. Suppose $\mathbf{w}^{f}=0$ for a non-constant $f \in C^{\infty}(M)$. Then we have

$$
\nabla f=\mathbf{w}(f) \mathbf{w}
$$

and taking covariant derivative with respect to $X \in \mathfrak{X}(M)$, we get

$$
\mathcal{H}_{f}(X)=X(\mathbf{w}(f)) \mathbf{w}+\mathbf{w}(f) \Psi X, \quad X \in \mathfrak{X}(M) .
$$

As the operator $\mathcal{H}_{f}$ is symmetric and $\Psi$ is skew-symmetric, we obtain

$$
2 \mathbf{w}(f) g(\Psi X, Y)=\alpha(X) Y(\mathbf{w}(f))-\alpha(Y) X(\mathbf{w}(f)), \quad X, Y \in \mathfrak{X}(M),
$$

that is,

$$
2 \mathbf{w}(f) \Psi X=\alpha(X) \nabla \mathbf{w}(f)-X(\mathbf{w}(f)) \mathbf{w} .
$$

Taking $X=\mathbf{w}$ in above equation and using Equation (7), we immediately get

$$
\nabla \mathbf{w}(f)=\mathbf{w} \mathbf{w}(f) \mathbf{w},
$$

then

$$
\|\nabla \mathbf{w}(f)\|^{2}=(\mathbf{w w}(f))^{2} .
$$

Now, taking a local orthonormal frame $\left\{e_{1}, \ldots, e_{n}\right\}, n=\operatorname{dim} M$ on $M$ and using it with Equation (14), we conclude

$$
\begin{gathered}
4(\mathbf{w}(f))^{2}\|\Psi\|^{2}=\sum_{i=1}^{n} g\left(2 \mathbf{w}(f) \Psi e_{i}, 2 \mathbf{w}(f) \Psi e_{i}\right) \\
=2\|\nabla \mathbf{w}(f)\|^{2}-2(\mathbf{w} \mathbf{w}(f))^{2} .
\end{gathered}
$$

Using Equation (15) in above equation, we get $(\mathbf{w}(f))^{2}\|\Psi\|^{2}=0$ and as $M$ is connected with $\mathbf{w}$ non-trivial $(\Psi \neq 0)$, we have $\mathbf{w}(f)=0$. This proves $\nabla f=0$ and we get a contradiction to the fact that $f$ is a non-constant function. Hence, $\mathbf{w}^{f} \neq 0$.

As seen in above Proposition, for each non-constant function $f \in C^{\infty}(M)$ on an $n$ dimensional connected $(M, g)$ that admits a non-trivial unit Killing vector field $\mathbf{w}$, the associated vector field $\mathbf{w}^{f}$ is non-zero vector field. In the next result, we show that if there exists a smooth non-constant function $f \in C^{\infty}(M)$ on an $n$-dimensional compact and connected $(M, g)$ that admits a non-trivial unit Killing vector field $\mathbf{w}$ satisfying $\Delta f=-n$ and the integral of the Ricci curvature $S\left(\mathbf{w}^{f}, \mathbf{w}^{f}\right)$ has certain lower bound is necessary and sufficient for $(M, g)$ to be isometric to the unit sphere $\mathbf{S}^{2 m+1}$, giving a characterization of the unit sphere $\mathbf{S}^{2 m+1}$.

Theorem 1. Let $\mathbf{w}$ be a non-trivial unit Killing vector field on an n-dimensional compact and connected Riemannian manifold $(M, g)$. Then there exists a non-constant function $f \in C^{\infty}(M)$ satisfying $\Delta f=-n f$ and

$$
\int_{M} S\left(\mathbf{w}^{f}, \mathbf{w}^{f}\right) \geq \int_{M}\left(2 \operatorname{Hess}(f)(\nabla \mathbf{w}(f), \mathbf{w})+n(n-1) f^{2}+(\mathbf{w}(f))^{2}\left(\|\Psi\|^{2}-2 n\right)\right),
$$


if and only if, $n$ is odd $(n=2 m+1))$ and $(M, g)$ is isometric to the unit sphere $\mathbf{S}^{2 m+1}$.

Proof. Let $\mathbf{w}$ be a non-trivial unit Killing vector field and $f \in C^{\infty}(M)$ be a non-constant function such that $\Delta f=-n f$. Then the associated vector field $\mathbf{w}^{f}$ is non-zero vector field and

$$
\mathbf{w}^{f}=\nabla f-\mathbf{w}(f) \mathbf{w} .
$$

Taking into account Equations (3) and (8), we have

$$
R\left(X_{1}, X_{2}\right) \nabla f=\left(\nabla \mathcal{H}_{f}\right)\left(X_{1}, X_{2}\right)-\left(\nabla \mathcal{H}_{f}\right)\left(X_{2}, X_{1}\right), \quad X_{1}, X_{2} \in \mathfrak{X}(M)
$$

and for a local orthonormal frame $\left\{e_{1}, \ldots, e_{n}\right\}$, we have

$$
\Delta f=\sum_{i=1}^{n} g\left(\mathcal{H}_{f}\left(e_{i}\right), e_{i}\right)
$$

Thus, on using Equation (17) and symmetry of the operator $\mathcal{H}_{f}$, we get

$$
\begin{gathered}
X(\Delta f)=\sum_{i=1}^{n} g\left(R\left(X, e_{i}\right) \nabla f+\left(\nabla \mathcal{H}_{f}\right)\left(e_{i}, X\right), e_{i}\right) \\
=-S(X, \nabla f)+g\left(X, \sum_{i=1}^{n}\left(\nabla \mathcal{H}_{f}\right)\left(e_{i}, e_{i}\right)\right), \quad X \in \mathfrak{X}(M) .
\end{gathered}
$$

Note that Equation (16) gives

$$
\nabla_{X} \mathbf{w}^{f}=\mathcal{H}_{f}(X)-X(\mathbf{w}(f)) \mathbf{w}-\mathbf{w}(f) \Psi X .
$$

Now, using Equations (18) and (19), we proceed to find $\operatorname{div}\left(\mathcal{H}_{f}\left(\mathbf{w}^{f}\right)\right)$ and get

$$
\begin{gathered}
\operatorname{div}\left(\mathcal{H}_{f}\left(\mathbf{w}^{f}\right)\right) \\
=\sum_{i=1}^{n} g\left(\mathcal{H}_{f}\left(e_{i}\right)-e_{i}(\mathbf{w}(f)) \mathbf{w}-\mathbf{w}(f) \Psi e_{i}, \mathcal{H}_{f}\left(e_{i}\right)\right) \\
+\mathbf{w}^{f}(\Delta f)+S\left(\mathbf{w}^{f}, \nabla f\right) .
\end{gathered}
$$

Integrating, we obtain

$$
\int_{M}\left(\left\|\mathcal{H}_{f}\right\|^{2}-\operatorname{Hess}(f)(\nabla \mathbf{w}(f), \mathbf{w})+S\left(\mathbf{w}^{f}, \nabla f\right)+\mathbf{w}^{f}(\Delta f)\right)=0,
$$

where we used $\operatorname{Tr} \mathcal{H}_{f} \Psi=0$. Using $\Delta f=-n f$ and Equation (16), we have $\mathbf{w}^{f}(\Delta f)=$ $-n\left\|\mathbf{w}^{f}\right\|^{2}$, as $\mathbf{w}$ and $\mathbf{w}^{f}$ are orthogonal and the above integral takes the form

$$
\int_{M}\left(\left\|\mathcal{H}_{f}\right\|^{2}-\operatorname{Hess}(f)(\nabla \mathbf{w}(f), \mathbf{w})+S\left(\mathbf{w}^{f}, \nabla f\right)-n\left\|\mathbf{w}^{f}\right\|^{2}\right)=0 .
$$

Next, we use Equation (6) to get

$$
\sum_{i=1}^{n}(\nabla \Psi)\left(e_{i}, e_{i}\right)=-T(\mathbf{w})
$$


and using this equation and Equation (19), in computing $\operatorname{div}\left(\mathbf{w}(f) \Psi\left(\mathbf{w}^{f}\right)\right)$, we reach at

$$
\begin{gathered}
\operatorname{div}\left(\mathbf{w}(f) \Psi\left(\mathbf{w}^{f}\right)\right)=g\left(\nabla \mathbf{w}(f), \Psi\left(\mathbf{w}^{f}\right)\right)+\mathbf{w}(f) \operatorname{div}\left(\Psi\left(\mathbf{w}^{f}\right)\right) \\
=g\left(\nabla \mathbf{w}(f), \Psi\left(\mathbf{w}^{f}\right)\right)+\mathbf{w}(f) S\left(\mathbf{w}^{f}, \mathbf{w}\right) \\
-\mathbf{w}(f) \sum_{i=1}^{n} g\left(\mathcal{H}_{f}\left(e_{i}\right)-e_{i}(\mathbf{w}(f)) \mathbf{w}-\mathbf{w}(f) \Psi e_{i}, \Psi e_{i}\right) .
\end{gathered}
$$

Use of $\operatorname{Tr} \mathcal{H}_{f} \Psi=0$, Equation (7), and outcome of Equation (16) as $\Psi\left(\mathbf{w}^{f}\right)=\Psi(\nabla f)$ in above equation yields

$$
\operatorname{div}\left(\mathbf{w}(f) \Psi\left(\mathbf{w}^{f}\right)\right)=g(\nabla \mathbf{w}(f), \Psi(\nabla f))+\mathbf{w}(f) S\left(\mathbf{w}^{f}, \mathbf{w}\right)+\mathbf{w}(f)^{2}\|\Psi\|^{2} .
$$

Also, we have $X(\mathbf{w}(f))=X g(\mathbf{w}, \nabla f)=g(\Psi X, \nabla f)+g\left(\mathbf{w}, \mathcal{H}_{f} X\right)$, i.e.,

$$
\Psi(\nabla f)=\mathcal{H}_{f}(\mathbf{w})-\nabla \mathbf{w}(f) .
$$

Thus, we have

$$
\begin{gathered}
\operatorname{div}\left(\mathbf{w}(f) \Psi\left(\mathbf{w}^{f}\right)\right) \\
=H e s s(f)(\nabla \mathbf{w}(f), \mathbf{w})-\|\nabla \mathbf{w}(f)\|^{2}+\mathbf{w}(f) S\left(\mathbf{w}^{f}, \mathbf{w}\right)+\mathbf{w}(f)^{2}\|\Psi\|^{2}
\end{gathered}
$$

and integrating above equation, we get

$$
\int_{M}\left(H \operatorname{ess}(f)(\nabla \mathbf{w}(f), \mathbf{w})-\|\nabla \mathbf{w}(f)\|^{2}+\mathbf{w}(f) S\left(\mathbf{w}^{f}, \mathbf{w}\right)+\mathbf{w}(f)^{2}\|\Psi\|^{2}\right)=0 .
$$

Subtracting this equation from Equation (20), while noting that

$$
S\left(\mathbf{w}^{f}, \nabla f\right)-\mathbf{w}(f) S\left(\mathbf{w}^{f}, \mathbf{w}\right)=S\left(\mathbf{w}^{f}, \mathbf{w}^{f}\right),
$$

we conclude

$$
\begin{gathered}
\int_{\mathbf{M}}\left(\left\|\mathcal{H}_{f}\right\|^{2}-2 \operatorname{Hess}(f)(\nabla \mathbf{w}(f), \mathbf{w})-n\left\|\mathbf{w}^{f}\right\|^{2}+\|\nabla \mathbf{w}(f)\|^{2}\right. \\
\left.+S\left(\mathbf{w}^{f}, \mathbf{w}^{f}\right)-\mathbf{w}(f)^{2}\|\Psi\|^{2}\right)=0 .
\end{gathered}
$$

On using $\Delta f=-n f$, and the fact that flow $\left\{\phi_{t}\right\}$ of $\mathbf{w}$ consists of isometries, we have $\Delta \mathbf{w}(f)=\mathbf{w}(\Delta f)=-n \mathbf{w}(f)$, and above equation changes to

$$
\begin{gathered}
\int_{\mathbf{M}}\left(\left\|\mathcal{H}_{f}\right\|^{2}-2 \operatorname{Hess}(f)(\nabla \mathbf{w}(f), \mathbf{w})-n\left\|\mathbf{w}^{f}\right\|^{2}+n \mathbf{w}(f)^{2}\right. \\
\left.+S\left(\mathbf{w}^{f}, \mathbf{w}^{f}\right)-\mathbf{w}(f)^{2}\|\Psi\|^{2}\right)=0 .
\end{gathered}
$$


Now, in above equation, we use $\left\|\mathbf{w}^{f}\right\|^{2}=\|\nabla f\|^{2}-\mathbf{w}(f)^{2}$ (Equation (16)), i.e.,

$$
\int_{\mathbf{M}}\left\|\mathbf{w}^{f}\right\|^{2}=\int_{M}\left(\|\nabla f\|^{2}-\mathbf{w}(f)^{2}\right)=\int_{M}\left(n f^{2}-\mathbf{w}(f)^{2}\right)
$$

to conclude

$$
\begin{array}{r}
\int_{\mathbf{M}}\left(\left\|\mathcal{H}_{f}\right\|^{2}-2 \operatorname{Hess}(f)(\nabla \mathbf{w}(f), \mathbf{w})-n^{2} f^{2}+2 n \mathbf{w}(f)^{2}\right. \\
\left.+S\left(\mathbf{w}^{f}, \mathbf{w}^{f}\right)-\mathbf{w}(f)^{2}\|\Psi\|^{2}\right)=0 .
\end{array}
$$

Also, using $\frac{1}{n}(\Delta f)^{2}=n f^{2}$, we get

$$
\begin{gathered}
\int_{\mathbf{M}}\left(\frac{1}{n}(\Delta f)^{2}-\left\|\mathcal{H}_{f}\right\|^{2}\right)=\int_{\mathbf{M}} S\left(\mathbf{w}^{f}, \mathbf{w}^{f}\right) \\
-\int_{M}\left(2 \operatorname{Hess}(f)(\nabla \mathbf{w}(f), \mathbf{w})+n(n-1) f^{2}+\mathbf{w}(f)^{2}\left(\|\Psi\|^{2}-2 n\right)\right)
\end{gathered}
$$

and using the bound in the statement for integral of $S\left(\mathbf{w}^{f}, \mathbf{w}^{f}\right)$, we obtain

$$
\int_{\mathbf{M}}\left(\frac{1}{n}(\Delta f)^{2}-\left\|\mathcal{H}_{f}\right\|^{2}\right) \geq 0
$$

Thus, by Schwarz's inequality, we get

$$
\mathcal{H}_{f}=\frac{\Delta f}{n} I=-f I
$$

for non-constant function $f$. Hence, $(M, g)$ is isometric to the unit sphere $\mathbf{S}^{n}$ (cf. $\left.[15,16]\right)$. Please note that on an even-dimensional compact Riemannian manifold of positive sectional curvature a Killing vector field must have a zero (cf. [17]). As the Killing vector field $\mathbf{w}$ is unit vector field does not have a zero, we get that $n$ is odd say $2 m+1$. Hence, $(M, g)$ is isometric to the unit sphere $\mathbf{S}^{2 m+1}$.

Conversely, there is a unit Killing vector field $\mathbf{w}$ on the unit sphere $\mathbf{S}^{2 m+1}$ and by Equations (12) and (13) there is a non-constant smooth function $f$ satisfying

$$
\mathcal{H}_{f}=-f I, \quad \Delta f=-(2 m+1) f \text {. }
$$

Now,

$$
\begin{aligned}
S\left(\mathbf{w}^{f}, \mathbf{w}^{f}\right) & =2 m\left\|\mathbf{w}^{f}\right\|^{2}=2 m\|\nabla f-\mathbf{w}(f) \mathbf{w}\|^{2} \\
& =2 m\left(\|\nabla f\|^{2}-\mathbf{w}(f)^{2}\right)
\end{aligned}
$$

and

$$
\begin{aligned}
& \operatorname{Hess}(f)(\nabla \mathbf{w}(f), \mathbf{w})=-f g(\nabla \mathbf{w}(f), \mathbf{w}) \\
& =-f \mathbf{w} \mathbf{w}(f)=-\left(\mathbf{w}(f \mathbf{w}(f))-\mathbf{w}(f)^{2}\right) .
\end{aligned}
$$

Hence, we have

$$
\int_{\mathbf{M}} S\left(\mathbf{w}^{f}, \mathbf{w}^{f}\right)=2 m \int_{M}\left(\|\nabla f\|^{2}-\mathbf{w}(f)^{2}\right)
$$


and using Lemma 1 and Equations (21), (22) and Lemma 2 as $\|\Psi\|^{2}=2 m$, we get the integral

$$
\int_{M}\left(2 \operatorname{Hess}(f)(\nabla \mathbf{w}(f), \mathbf{w})+2 m(2 m+1) f^{2}+\mathbf{w}(f)^{2}\left(\|\Psi\|^{2}-2(2 m+1)\right)\right.
$$

is equal to

$$
\int_{M}\left(2 \mathbf{w}(f)^{2}+2 m\|\nabla f\|^{2}-(2 m+2) \mathbf{w}(f)^{2}\right)
$$

where we used

$$
\int_{M}\|\nabla f\|^{2}=(2 m+1) \int_{M} f^{2} .
$$

Thus, by Equations (21), (23) and (24) we see that all requirements of the statement are met.

Remark 1. If the equality in the statement of Theorem 1 holds, then following the proof of the Theorem 1, we conclude

$$
\int_{M}\left(\frac{1}{n}(\Delta f)^{2}-\left\|\mathcal{H}_{f}\right\|^{2}\right)=0
$$

Using the Schwarz's inequality in above equation, we get

$$
\mathcal{H}_{f}=\frac{\Delta f}{n} I=-f I
$$

and we get the same conclusion as in Theorem 1.

We would like to point that a similar situation is considered in [18].

In the next result we show that for positive constant $c$, if there is a smooth function $f \in C^{\infty}(M)$ satisfying $\Delta f=-n c f$ on an $n$-dimensional compact and connected $(M, g)$ that admits a non-trivial unit Killing vector field $\mathbf{w}$ with $S(\mathbf{w}, \mathbf{w}) \leq(n-1) c$, and the Ricci curvature $S(\nabla f-\mathbf{w}, \nabla f-\nabla \mathbf{w})$ has certain lower bound, is necessary and sufficient for $(M, g)$ to be isometric to the sphere $\mathbf{S}^{2 m+1}(c)$, giving a characterization of the odddimensional sphere $\mathbf{S}^{2 m+1}(c)$.

Theorem 2. Let $\mathbf{w}$ be a non-trivial unit Killing vector field on an n-dimensional compact and connected Riemannian manifold $(M, g)$. Then there exists a non-constant function $f \in C^{\infty}(M)$ satisfying $\Delta f=-n c f$ for a constant $c>0,\|\Psi\|^{2} \leq(n-1) c$ and the Ricci curvature in the direction of the vector field $\nabla f-\mathbf{w}$ is bounded below by $(n-1) c$, if and only if, $n$ is odd $(n=2 m+1))$ and $(M, g)$ is isometric to the sphere $\mathbf{S}^{2 m+1}(c)$.

Proof. Let $\mathbf{w}$ be a non-trivial unit Killing vector field on $(M, g)$ such that $\|\Psi\|^{2} \leq(n-1) c$ and $f \in C^{\infty}(M)$ be a non-constant function such that $\Delta f=-n c f$ and

$$
S(\nabla f-\mathbf{w}, \nabla f-\mathbf{w}) \geq(n-1) c\|\nabla f-\mathbf{w}\|^{2}, \quad c>0 .
$$

We have $S(\nabla f-\mathbf{w}, \nabla f-\mathbf{w})=S(\nabla f, \nabla f)-2 S(\nabla f, \mathbf{w})+S(\mathbf{w}, \mathbf{w})$, using Lemmas 2 and 3 , we get

$$
\int_{\mathbf{M}} S(\nabla f-\mathbf{w}, \nabla f-\mathbf{w})=\int_{\mathbf{M}}\left(S(\nabla f, \nabla f)+\|\Psi\|^{2}\right) .
$$

Applying the Bochner's formula

$$
\int_{\mathbf{M}}\left(S(\nabla f, \nabla f)+\left\|\mathcal{H}_{f}\right\|^{2}-(\Delta f)^{2}\right)=0
$$


in Equation (26), we obtain

$$
\int_{\mathbf{M}} S(\nabla f-\mathbf{w}, \nabla f-\mathbf{w})=\int_{\mathbf{M}}\left((\Delta f)^{2}-\left\|\mathcal{H}_{f}\right\|^{2}+\|\Psi\|^{2}\right) .
$$

Also, we have $\|\nabla f-\mathbf{w}\|^{2}=\|\nabla f\|^{2}+\mathbf{1}-2 \mathbf{w}(f)$ and using the outcome of $\Delta f=-n c f$, namely

$$
\int_{\mathbf{M}}\|\nabla f\|^{2}=n c \int_{\mathbf{M}} f^{2}
$$

and Lemma 1, we get

$$
\int_{\mathbf{M}}\|\nabla f-\mathbf{w}\|^{2}=\int_{\mathbf{M}}\left(1+n c f^{2}\right) .
$$

Using above equation and Equation (27), we conclude

$$
\begin{array}{r}
\int_{\mathbf{M}}\left(S(\nabla f-\mathbf{w}, \nabla f-\mathbf{w})-(n-1) c\|\nabla f-\mathbf{w}\|^{2}\right)=\int_{\mathbf{M}}\left((\Delta f)^{2}-\left\|\mathcal{H}_{f}\right\|^{2}+\|\Psi\|^{2}\right) \\
-(n-1) c \int_{\mathbf{M}}\left(1+n c f^{2}\right) .
\end{array}
$$

Using $\Delta f=-n c f$, we get

$$
\begin{aligned}
\int_{\mathbf{M}}\left(S(\nabla f-\mathbf{w}, \nabla f-\mathbf{w})-(n-1) c\|\nabla f-\mathbf{w}\|^{2}\right)= & \int_{\mathbf{M}}\left(\frac{1}{n}(\Delta f)^{2}-\left\|\mathcal{H}_{f}\right\|^{2}\right) \\
& +\int_{\mathbf{M}}\left(\|\Psi\|^{2}-(n-1) c\right) .
\end{aligned}
$$

Now, using $\|\Psi\|^{2} \leq(n-1) c$, we see that

$$
\int_{\mathbf{M}}\left(S(\nabla f-\mathbf{w}, \nabla f-\mathbf{w})-(n-1) c\|\nabla f-\mathbf{w}\|^{2}\right) \leq \int_{\mathbf{M}}\left(\frac{1}{n}(\Delta f)^{2}-\left\|\mathcal{H}_{f}\right\|^{2}\right)
$$

and using inequality (25) in above inequality, we have

$$
0 \leq \int_{\mathbf{M}}\left(\frac{1}{n}(\Delta f)^{2}-\left\|\mathcal{H}_{f}\right\|^{2}\right)
$$

Thus, in view of Schwarz's inequality, we obtain

$$
\mathcal{H}_{f}=\frac{\Delta f}{n} I=-c f I,
$$

that is, $(M, g)$ is isometric to the sphere $\mathbf{S}^{n}(c)$. As in the proof of Theorem 1 , we get that $n$ is odd say $2 m+1$ and $(M, g)$ is isometric to the sphere $\mathbf{S}^{2 m+1}(c)$. Converse trivially follows using Equations (10)-(13), Lemma 2 and expression for Ricci tensor of the sphere $\mathbf{S}^{2 m+1}(c)$.

\section{Killing Vector Fields and Sasakian Manifolds}

Recall that a $(2 m+1)$-dimensional manifold $M$ is said to have an almost contact metric structure $(\phi, \xi, \eta, g)$, if

$$
\phi^{2}=-I+\eta \otimes \xi, \phi \xi=0, \quad \eta \circ \phi=0, \quad g\left(\phi X_{1}, \phi X_{2}\right)=g\left(X_{1}, X_{2}\right)-\eta\left(X_{1}\right) \eta\left(X_{2}\right),
$$


where $\phi$ is a skew-symmetric $(1,1)$ tensor field, $\xi$ is a unit vector field, $\eta$ is 1 -form dual to $\xi$ and $g$ is the Riemannian metric on $M$ (see [12]). An almost contact metric manifold $M(\phi, \xi, \eta, g)$ is said to be a contact metric manifold if

$$
d \eta\left(X_{1}, X_{2}\right)=g\left(X_{1}, \phi X_{2}\right) .
$$

If unit vector field $\xi$ of the contact metric manifold $M(\phi, \xi, \eta, g)$ is Killing, then it is said to be a K-contact manifold. Also, a contact metric manifold $M(\phi, \xi, \eta, g)$ is said to be a Sasakian manifold if

$$
(\nabla \phi)\left(X_{1}, X_{2}\right)=\eta\left(X_{2}\right) X_{1}-g\left(X_{1}, X_{2}\right) \xi .
$$

Given a unit Killing vector field $\mathbf{w}$ on an $n$-dimensional Riemannian manifold $(M, g)$ there is naturally associated a skew-symmetric operator $\phi=-\Psi$ on $(M, g)$ and there is a smooth 1-form $\alpha$ dual to $\mathbf{w}$. A natural question is when does $(\phi, \mathbf{w}, \alpha, g)$ become a K-contact structure on $M$ ? Of course, it requires $n$ to be odd, $\phi$ should satisfy relation similar to almost contact metric structure, namely

$$
\phi^{2}=-I+\alpha \otimes \mathbf{w}, g\left(\phi X_{1}, \phi X_{2}\right)=g\left(X_{1}, X_{2}\right)-\alpha\left(X_{1}\right) \alpha\left(X_{2}\right), \quad X_{1}, X_{2} \in \mathfrak{X}(M)
$$

and other requirements are automatically met by Equations (2) and (7) (cf. [12,13]). In this section, we answer this question by showing that $(\phi, \mathbf{w}, \alpha, g)$ becomes a K-contact structure on $M$, if and only if, the Riemannian manifold $(M, g)$ admitting the unit Killing vector field $\mathbf{w}$ with sectional curvatures of plane sections containing $\mathbf{w}$ are constant equal to 1 . We also find conditions under which a Riemannian manifold $(M, g)$ admitting a unit vector field $\mathbf{w}$ is an Einstein-Sasakian manifold.

Theorem 3. Let $\mathbf{w}$ be a unit Killing vector field on an n-dimensional connected Riemannian manifold $(M, g)$. Then $(\phi, \mathbf{w}, \alpha, g)$ is a K-contact structure, if and only if, sectional curvatures of the plane sections containing $\mathbf{w}$ are constant equal to 1 .

Proof. Let $\mathbf{w}$ be a unit Killing vector field on $(M, g)$ and $\alpha$ be smooth 1-form dual to $\mathbf{w}$. Suppose the sectional curvature $K(\Pi)=1$ for a plane section $\Pi$ containing $\mathbf{w}$. Then, we have

$$
R(X, \mathbf{w} ; \mathbf{w}, X)=\|X\|^{2}-\alpha(X)^{2}, \quad X \in \mathfrak{X}(M),
$$

where $R\left(X_{1}, X_{2} ; X_{3}, X_{4}\right)=g\left(R\left(X_{1}, X_{2}\right) X_{3}, X_{4}\right)$. Polarizing Equation (28), we get

$$
R\left(X_{1}, \mathbf{w} ; \mathbf{w}, X_{2}\right)+R\left(X_{2}, \mathbf{w} ; \mathbf{w}, X_{1}\right)=2 g\left(X_{1}, X_{2}\right)-2 \alpha\left(X_{1}\right) \alpha\left(X_{2}\right),
$$

that is,

$$
R\left(X_{1}, \mathbf{w} ; \mathbf{w}, X_{2}\right)=g\left(X_{1}, X_{2}\right)-\alpha\left(X_{1}\right) \alpha\left(X_{2}\right) .
$$

This proves that

$$
R(X, \mathbf{w}) \mathbf{w}=X-\alpha(X) \mathbf{w}, \quad X \in \mathfrak{X}(M) .
$$

Now, using Equations (6) and (7), we get

$$
R(X, \mathbf{w}) \mathbf{w}=(\nabla \Psi)(X, \mathbf{w})=-\Psi^{2} X,
$$

and combining above equation with $\phi=-\Psi$ and Equation (29), we conclude

$$
\phi^{2}=-I+\alpha \otimes \mathbf{w} .
$$

Also, we have

$$
g\left(\phi X_{1}, \phi X_{2}\right)=g\left(X_{1}, X_{2}\right)-\alpha\left(X_{1}\right) \alpha\left(X_{2}\right), \quad X_{1}, X_{2} \in \mathfrak{X}(M) .
$$


According to (2), we have

$$
\begin{gathered}
d \alpha\left(X_{1}, X_{2}\right)=\frac{1}{2}\left\{g\left(\nabla_{X_{1}} \mathbf{w}, X_{2}\right)-g\left(\nabla_{X_{2}} \mathbf{w}, X_{1}\right)\right\} \\
=\frac{1}{2}\left\{g\left(\Psi X_{1}, X_{2}\right)-g\left(\Psi X_{2}, X_{1}\right)\right\} \\
=-g\left(X_{1}, \Psi X_{2}\right)=g\left(X_{1}, \phi X_{2}\right), \quad X_{1}, X_{2} \in \mathfrak{X}(M) .
\end{gathered}
$$

Hence, $(\phi, \mathbf{w}, \alpha, g)$ is a K-contact structure on $M$.

The converse is obvious (cf. [12,13]).

In [3], it was observed that presence of Killing vector field of constant length on $(M, g)$ with certain sectional curvatures positive and Ricci operator parallel implies $(M, g)$ is an Einstein manifold. In the next result, we find conditions under which a compact Riemannian manifold admitting a unit Killing vector field is an Einstein manifold.

Theorem 4. Let $\mathbf{w}$ be a non-trivial unit Killing vector field on an n-dimensional compact and connected Riemannian manifold $(M, g), n>2$ with section curvatures of plane sections containing $\mathbf{w}$ being positive. If the following conditions are satisfied

$$
\text { (i) }(\nabla T)(X, \mathbf{w})=(\nabla T)(\mathbf{w}, X), \quad X \in \mathfrak{X}(M), \quad \text { (ii) } \quad\|\Psi\|^{4}+\operatorname{Tr}\left(T \circ \Psi^{2}\right) \geq 0,
$$

then $(M, g)$ is an Einstein manifold.

Proof. Let $\mathbf{w}$ be a non-trivial unit Killing vector field on a compact and connected $(M, g)$. Suppose that $K(\Pi)>0$ for a plane section $\Pi$ containing $\mathbf{w}$ and the following conditions hold

$$
(\nabla T)(X, \mathbf{w})=(\nabla T)(\mathbf{w}, X), \quad X \in \mathfrak{X}(M), \quad\|\Psi\|^{4}+\operatorname{Tr}\left(T \circ \Psi^{2}\right) \geq 0 .
$$

Using Lemma 2, we define a vector field $\mathbf{u}$ orthogonal to $\mathbf{w}$ by

$$
T(\mathbf{w})=\mathbf{u}+\|\Psi\|^{2} \mathbf{w} .
$$

Taking covariant derivative in above equation with respect to $X \in \mathfrak{X}(M)$, we get

$$
(\nabla T)(X, \mathbf{w})+T(\Psi X)=\nabla_{X} \mathbf{u}+X\left(\|\Psi\|^{2}\right) \mathbf{w}+\|\Psi\|^{2} \Psi X .
$$

As the flow of $\mathbf{w}$ consists of isometries of $(M, g)$, we have $\left(£_{\mathbf{w}} T\right)=0$, i.e.,

$$
(\nabla T)(\mathbf{w}, X)=\Psi T(X)-T(\Psi X), \quad X \in \mathfrak{X}(M)
$$

and using it with the first equation in Equation (30), we have

$$
(\nabla T)(X, \mathbf{w})=\Psi T(X)-T(\Psi X), \quad X \in \mathfrak{X}(M) .
$$

Thus, using Equation (33) in Equation (32), we conclude

$$
\nabla_{X} \mathbf{u}=\Psi T(X)-\|\Psi\|^{2} \Psi X-X\left(\|\Psi\|^{2}\right) \mathbf{w}, \quad X \in \mathfrak{X}(M) .
$$

Taking the inner product with $\mathbf{w}$ in above equation and using Equation (7) and $g(\mathbf{u}, \mathbf{w})=0$, we obtain

$$
-g(\mathbf{u}, \Psi X)=-X\left(\|\Psi\|^{2}\right),
$$


that is, the gradient $\nabla\|\Psi\|^{2}$ is given by

$$
\nabla\|\Psi\|^{2}=-\Psi \mathbf{u} .
$$

Using Equation (33) and a local orthonormal frame $\left\{e_{1}, \ldots, e_{n}\right\}$ on $M$, we compute the $\operatorname{div}(\Psi \mathbf{u})$ and get the following

$$
\operatorname{div}(\Psi \mathbf{u})=\sum_{i=1}^{n} g\left(\nabla_{e_{i}} \Psi \mathbf{u}, e_{i}\right)=\sum_{i=1}^{n} g\left((\nabla \Psi)\left(e_{i}, \mathbf{u}\right)+\Psi \nabla_{e_{i}} \mathbf{u}, e_{i}\right)
$$

and using Equations (6) and (34) and Lemma 2, we conclude

$$
\operatorname{div}(\Psi \mathbf{u})=\|\mathbf{u}\|^{2}+\|\Psi\|^{4}+\operatorname{Tr}\left(T \circ \Psi^{2}\right) .
$$

Integrating above equation and using the inequality in Equation (30), we have $\mathbf{u}=0$ and consequently, Equation (35) implies that $\|\Psi\|^{2}$ is a constant. Hence, Equation (31) implies that $T(\mathbf{w})=c \mathbf{w}$, where $c$ is a positive constant because $\mathbf{w}$ is non-trivial Killing vector field. Taking covariant derivative in the equation $T(\mathbf{w})=c \mathbf{w}$ while using Equations (2) and (33), we get

$$
\Psi T(X)=c \Psi X, \quad X \in \mathfrak{X}(M),
$$

that is,

$$
\Psi(T(X)-c X)=0, \quad X \in \mathfrak{X}(M) .
$$

Now, using Equations (6) and (7), we have $R(X, \mathbf{w}) \mathbf{w}=(\nabla \Psi)(X, \mathbf{w})=-\Psi^{2} X$, i.e.,

$$
R(X, \mathbf{w} ; \mathbf{w}, X)=\|\Psi X\|^{2}, \quad X \in \mathfrak{X}(M) .
$$

Please note that using $T(\mathbf{w})=c \mathbf{w}$, we get $g(T(X)-c X, \mathbf{w})=0$, i.e., $T(X)-c X$ is orthogonal to $\mathbf{w}$ for all $X \in \mathfrak{X}(M)$. Thus, using Equations (36) and (37), we get that the sectional curvature

$$
R(T X-c X, \mathbf{w} ; \mathbf{w}, T X-c Z)=0
$$

and the condition in the statement that $K(\Pi)>0$ for a plane section $\Pi$ containing $\mathbf{w}$ implies $T(X)=c X$ for all $X \in \mathfrak{X}(M)$. Hence, $(M, g)$ is an Einstein manifold.

We can bypass the requirements that manifold is compact and the condition (ii) in Theorem 4 by assuming certain vector field is parallel as seen in the following:

Theorem 5. Let $\mathbf{w}$ be a non-trivial unit Killing vector field on an n-dimensional complete and connected Riemannian manifold $(M, g), n>2$ with section curvatures of plane sections containing $\mathbf{w}$ being positive. If the vector field $T(\mathbf{w})-\|\Psi\|^{2} \mathbf{w}$ is parallel and the Ricci operator $T$ satisfies

$$
(\nabla T)(X, \mathbf{w})=(\nabla T)(\mathbf{w}, X), \quad X \in \mathfrak{X}(M),
$$

then $(M, g)$ is a compact Einstein manifold.

Proof. Follow the proof of Theorem 4 up to Equation (35) and use that the vector field $\mathbf{u}=T(\mathbf{w})-\|\Psi\|^{2} \mathbf{w}$ is parallel, in view of Equation (34), we get

$$
\Psi\left(T(X)-\|\Psi\|^{2} X\right)=X\left(\|\Psi\|^{2}\right) \mathbf{w}, \quad X \in \mathfrak{X}(M) .
$$

Taking the inner product with $\mathbf{w}$ in above equation and using Equation (7), we have $X\left(\|\Psi\|^{2}\right)=0, X \in \mathfrak{X}(M)$, and we conclude $\|\Psi\|^{2}$ is a constant $c>0$ (as $\mathbf{w}$ is a non-trivial Killing vector field). Then Equation (35) implies $\Psi(\mathbf{u})=0$, which gives $R(\mathbf{u}, \mathbf{w} ; \mathbf{w}, \mathbf{u})=\|\Psi \mathbf{u}\|^{2}=0$ and as the sectional curvatures of plane sections containing $\mathbf{w}$ are positive, we get $\mathbf{u}=0$. Following Proof of Theorem 4 , we get that $(M, g)$ is an Einstein 
manifold. Please note that as $S(X, X)=c\|X\|^{2}$ and $c>0$, the Ricci curvature is strictly positive and therefore by Myer's Theorem $(M, g)$ is compact.

Remark 2. We give two examples, one satisfying conditions in Theorems 4 and 5 and other does not obeying conditions in these theorems.

Example 1. Consider the sphere $\mathbf{S}^{2 m+1}(c)$. We have seen in the Preliminaries that $\mathbf{S}^{2 m+1}(c)$ admits a unit Killing vector field $\mathbf{w}=-J \zeta$, where $\zeta$ is the unit normal and $J$ is the complex structure on the complex space $\mathbf{C}^{m+1}$. Moreover, we have (see Equation (10))

$$
\nabla_{X} \mathbf{w}=\Psi X, \quad X \in \mathfrak{X}\left(\mathbf{S}^{2 m+1}(c)\right),
$$

where $\Psi X=-\sqrt{c}(J X)^{T}$, where $(J X)^{T}$ is the tangential component of $J X$ to $\mathbf{S}^{2 m+1}(c)$. The Ricci operator $T$ of $\mathbf{S}^{2 m+1}(c)$ is given by

$$
T(X)=2 m c X, \quad X \in \mathfrak{X}\left(\mathbf{S}^{2 m+1}(c)\right),
$$

which clearly satisfies

$$
(\nabla T)(X, \mathbf{w})=(\nabla T)(\mathbf{w}, X) .
$$

Also, on taking a local orthonormal frame $\left\{e_{1}, \ldots, e_{2 m+1}\right\}$ on $\mathbf{S}^{2 m+1}(c)$, we have

$$
\begin{gathered}
\|\Psi\|^{2}=\sum_{i=1}^{2 m+1} g\left(\Psi e_{i}, \Psi e_{i}\right)=\sum_{i=1}^{2 m+1} c g\left(\left(J e_{i}\right)^{T},\left(J e_{i}\right)^{J}\right)= \\
c \sum_{i=1}^{2 m+1}\left\langle J e_{i}-\left\langle J e_{i}, \zeta\right\rangle \zeta, J e_{i}-\left\langle J e_{i}, \zeta\right\rangle \zeta\right\rangle=2 m c
\end{gathered}
$$

and

$$
\begin{gathered}
\operatorname{Tr}\left(T \circ \Psi^{2}\right)=\sum_{i=1}^{2 m+1} g\left(T\left(\Psi^{2} e_{i}\right), e_{i}\right)=2 m c \sum_{i=1}^{2 m+1} g\left(\Psi^{2} e_{i}, e_{i}\right)= \\
-2 m c\|\Psi\|^{2}=-4 m^{2} c^{2} .
\end{gathered}
$$

Consequently, we have

$$
\|\Psi\|^{4}+\operatorname{Tr}\left(T \circ \Psi^{2}\right)=0 .
$$

Moreover, we have $T(\mathbf{w})-\|\Psi\|^{2} \mathbf{w}=2 m c \mathbf{w}-2 m c \mathbf{w}=0$, i.e., $T(\mathbf{w})-\|\Psi\|^{2} \mathbf{w}$ is parallel. Hence, we see that the conditions (i) and (ii) in Theorem 4, as well as both conditions in Theorem 5 hold for the Einstein manifold $\mathbf{S}^{2 m+1}(c)$ with unit Killing vector field $\mathbf{w}$.

Example 2. Consider the 3-dimensional ellipsoid $M^{3}$ defined by

$$
M^{3}=\left\{u \in \mathbf{E}^{4}: u_{1}^{2}+u_{2}^{2}+a u_{3}^{2}+a u_{4}^{2}=1\right\},
$$

where constant $a>0$ and $u_{1}, \ldots, u_{4}$ are Euclidean coordinates on $\mathbf{E}^{4}$. Then $\left(M^{3}, g\right)$ is a compact 3-dimensional Riemannian manifold with $g$ the induced metric as a hypersurface of the Euclidean space $\mathbf{E}^{4}$. As seen in the Preliminaries there is a unit Killing vector field $\mathbf{w}$ on $\left(M^{3}, g\right)$ given by

$$
\mathbf{w}=\left(-u_{2}, u_{1},-\sqrt{a} u_{4}, \sqrt{a} u_{3}\right) .
$$

We claim that $\left(M^{3}, g\right)$ is not an Einstein manifold. Suppose $\left(M^{3}, g\right)$ is an Einstein manifold with Ricci tensor and Ricci operator given by

$$
S=\frac{\tau}{3} g, \quad T=\frac{\tau}{3} I .
$$


Please note that the curvature tensor field $R$ of the 3-dimensional $\left(M^{3}, g\right)$ is given by

$$
\begin{gathered}
R\left(X_{1}, X_{2}\right) X_{3}=S\left(X_{2}, X_{3}\right) X_{1}-S\left(X_{1}, X_{3}\right) X_{2}+g\left(X_{2}, X_{3}\right) T\left(X_{1}\right)-g\left(X_{1}, X_{3}\right) T\left(X_{2}\right)- \\
\frac{\tau}{3}\left\{g\left(X_{2}, X_{3}\right) X_{1}-g\left(X_{1}, X_{3}\right) X_{2}\right\},
\end{gathered}
$$

that is,

$$
R\left(X_{1}, X_{2}\right) X_{3}=\frac{\tau}{6}\left\{g\left(X_{2}, X_{3}\right) X_{1}-g\left(X_{1}, X_{3}\right) X_{2}\right\} .
$$

This shows that $\left(M^{3}, g\right)$ is space of constant curvature $\frac{\tau}{6}$ that is a contradiction. Hence, $\left(M^{3}, g\right)$ is not an Einstein manifold.

Using Theorem 3 and the condition on Ricci operator being a Codazzi type tensor as in Theorem 5, we get the following result, similar to Theorem 4.1 in [19].

Corollary 2. Let $\mathbf{w}$ be a non-trivial unit Killing vector field on an $n$-dimensional complete and connected Riemannian manifold $(M, g), n>2$ with section curvatures of plane sections containing $\mathbf{w}$ being constant equal to 1 . If the Ricci operator $T$ satisfies

$$
(\nabla T)(X, \mathbf{w})=(\nabla T)(\mathbf{w}, X), \quad X \in \mathfrak{X}(M),
$$

then $(M, g)$ is an Einstein Sasakian manifold.

Proof. By Theorem 3, we get that $(M, g)$ is a K-contact manifold, $n=2 m+1$ and $\|\Psi\|^{2}=$ $2 m$. Thus, $\|\Psi\|^{2}$ is a constant and by Equation (35), we get $\mathbf{u}=0$ and we get on similar lines as in proof of Theorem 5 that $(M, g)$ is an Einstein K-contact manifold with $S=2 m g$. Since Ricci curvature is strictly positive we get $(M, g)$ is compact. Hence, $(M, g)$ is an Einstein Sasakian manifold (cf. [13]).

Author Contributions: Conceptualization and methodology, S.D.; formal analysis, O.B.; writingoriginal draft preparation, S.D. and O.B.; writing-review and editing, S.D. and O.B.; supervision, S.D. and O.B.; project administration. All authors have read and agreed to the published version of the manuscript.

Funding: This research received no external funding.

Institutional Review Board Statement: Not applicable.

Informed Consent Statement: Not applicable.

Data Availability Statement: Not applicable.

Acknowledgments: The authors extend their appreciations to the Deanship of Scientific Research, King Saud University for funding this work through research group No (RG-1441-P182).

Conflicts of Interest: The authors declare no conflicts of interest.

\section{References}

1. Alodan, H.; Deshmukh, S.; Turki, N.; Vilcu, G. E. Hypersurfaces of a Sasakian manifold. Mathematics 2020, 8, 877. [CrossRef]

2. Berestovskii, V.; Nikonorov, Y. Killing vector fields of constant length on Riemannian manifolds. Sib. Math. J. 2008, 49, 395-407. [CrossRef]

3. Deshmukh, S. Characterizations of Einstein manifolds and odd-dimensional spheres. J. Geom. Phys. 2011, 61, 2058-2063. [CrossRef]

4. Deshmukh, S. A note on compact hypersurfaces in a Euclidean space. C. R. Math. Acad. Sci. Paris 2012, 350, 971-974. [CrossRef]

5. Deshmukh, S. Jacobi-type vector fields and Ricci soliton. Bull. Math. Soc. Sci. Math. Roum. 2012, 55, 41-50.

6. Deshmukh, S. Characterizing spheres and Euclidean spaces by conformal vector field. Ann. Mat. Pura Appl. 2017, 196, 2135-2145. [CrossRef]

7. Deshmukh, S.; Khan, V.A. Geodesic vector fields and eikonal equation on a Riemannian manifold. Indag. Math. 2019, 30, 542-552. [CrossRef]

8. Lynge, W.C. Sufficient conditions for periodicity of a Killing vector field. Proc. Amer. Math. Soc. 1973, 38, 614-616. [CrossRef] 
9. Rong, X. Positive curvature, local and global symmetry, and fundamental groups. Am. J. Math. 1999, 121, 931-943. [CrossRef]

10. Yorozu, S. Killing vector fields on complete Riemannian manifolds. Proc. Am. Math. Soc. 1982, 84, 115-120. [CrossRef]

11. Shore, S.N. Astrophysical Hydrodynamics: An Introduction; Wiley-VCH Verlag GmbH \& KGaA: Weinheim, Germany, 2007.

12. Blair, D.E. Contact Manifolds in Riemannian Geometry; Lectures Notes in Mathematics; Springer: Berlin, Germany; New York, NY, USA, 1976; Volume 509, pp. 395-407. .

13. Boyer, C.; Galicki, K. Sasakian Geometry. Oxford Mathematical Monographs; Oxford University Press: Oxford, NY, USA, 2008.

14. Deshmukh, S.; Al-Dayel, I.; Belova, O. A remarkable propery of concircular vector fields on a Riemannian manifold. Mathematics 2020, 8, 469. [CrossRef]

15. Obata, M. Conformal transformations of Riemannian manifolds. J. Differ. Geom. 1970, 4, 311-333. [CrossRef]

16. Obata, M. The conjectures on conformal transformations of Riemannian manifolds. J. Differ. Geom. 1971, 6, 247-258. [CrossRef]

17. do Carmo, M.P. Riemannian Geometry; Translated from the Second Portuguese Edition by Francis Flaherty; Mathematics: Theory \& Applications; Birkhäuser Boston, Inc.: Boston, MA, USA, 1992.

18. Crasmareanu, M. A new approach to gradient Ricci solitons and generalizations. Filomat 2019, 32, 3337-3346. [CrossRef]

19. Deshmukh, S.; Ishan, A. A note on contact metric manifolds. Hacet. J. Math. Stat. 2020, 49, 2007-2016. [CrossRef] 